\title{
Protein-water Dynamics in Antifreeze Protein III Activity
}

\author{
Yao Xu ${ }^{\mathrm{a}}$, Alexander Bäumer ${ }^{\mathrm{a}}$, Konrad Meister ${ }^{\mathrm{a}}$, Connor Bischak ${ }^{\mathrm{a}^{*}}$, Arthur L. DeVries ${ }^{\mathrm{c}}$,
} David M. Leitner ${ }^{\mathrm{b}}$ and Martina Havenith ${ }^{\mathrm{a}}$

\footnotetext{
${ }^{\text {a }}$ Lehrstuhl für Physikalische Chemie II, Ruhr Universität, 44801 Bochum, Germany;

${ }^{\mathrm{b}}$ Department of Chemistry, University of Nevada, Reno, NV 89557;

${ }^{\mathrm{c}}$ Department of Animal Biology, University of Illinois at Urbana-Champaign, Urbana, IL 61801

* Current address: Department of Chemistry, University of California, Berkeley, CA 94720
}

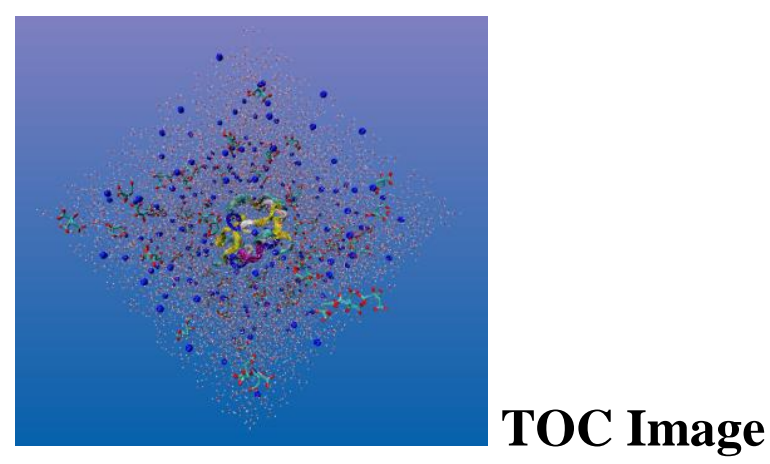

\begin{abstract}
We combine Terahertz absorption spectroscopy (THz) and molecular dynamics (MD) simulations to investigate the underlying molecular mechanism for the antifreeze activity of one class of antifreeze protein, antifreeze protein type III (AFP-III) with a focus on the collective water hydrogen bond dynamics near the protein. After summarizing our previous work on AFP-II, we present a new investigation of the effects of cosolutes on protein antifreeze activity by adding sodium citrate to the protein solution of AFP-III. Our results reveal that for AFP-III, unlike some other AFPs, the addition of the osmolyte sodium citrate does not affect the hydrogen bond dynamics at the protein surface significantly, as indicated by concentration dependent $\mathrm{THz}$ measurements. The present data, in combination with our previous $\mathrm{THz}$ measurements and molecular simulations, confirm that while long-range solvent perturbation is a necessary condition for the antifreeze activity of AFP-III, the local binding affinity determines the size of the hysteresis.
\end{abstract}




\section{INTRODUCTION}

Antifreeze proteins (AFPs) are a class of polypeptides widely distributed in certain plants, vertebrates, fungi and bacteria that permit their survival in subzero environments [1]. AFPs exhibit a unique ability to lower the temperature at which an ice crystal grows, as the temperature is depressed below the equilibrium melting/freezing point to a lower, hysteric freezing point [2]. Various fields [3] have witnessed the applications of AFPs, ranging from cold avoidance in plant biology [4] to cryosurgery in medicine [5]. However, details of the molecular mechanism how AFPs work are not yet fully understood. The generally accepted mechanism for the antifreeze activity is adsorption-inhibition [6-9], which proposes that AFPs adsorb onto the preferred growth sites of ice surface, thereby preventing ice growth in the normal low radius of curvature and restricting growth to regions between the adsorbed AFPs in a high radius of curvature front, and hence leading to the prevention of new ice growth until the temperature is lowered to the hysteretic freezing point, according to the Kelvin effect $[2,9,10]$.

AFPs show their diversity predominantly in fish, plant, insect, and sea ice organisms. Although there might be a consensus on the mechanism of the antifreeze activity in one category of organisms, there may not be a single unified mechanism for AFPs for all organisms. Nature has realized a broad variety of structural motifs for antifreeze proteins. As a matter of fact, more and more accumulated observations $[11,12,15,23,30,32]$ indicate that there exists distinct mechanisms, possibly even within a single category. For example, there are basically five types of AFPs in fish: antifreeze glycoproteins (AFGP) and Type I to IV AFPs. Different types of fish AFPs come from different types of fish with protein sizes ranging from $2.5 \mathrm{kDa}$ to $32 \mathrm{kDa}$. An early study [7] on type I AFP pointed to a local interaction of the protein with the nano ice crystal via 
a strong hydrogen bond interaction between ice and a hydroxyl group of the AFP. However, an overview on the systematic studies of several mutants of the protein did not support the anticipated decrease in antifreeze activity [8]. In a recent X-ray crystallographic picture of the AFP-ice interaction [9], Davies and coworkers found that an ice-like surface water structure is anchored via hydrogen bonds to the polypeptide backbone. These anchored waters are thought to facilitate the AFP interaction with ice [10]. Molecular Dynamics (MD) simulations revealed a gradient of hydrogen bond dynamics towards the ice-binding site for solvated proteins, with a more pronounced retardation of water hydrogen dynamics at the ice binding site [11].

THz spectroscopy probes large amplitude motions and the hydration of proteins, particularly the long-range and fast dynamics of collective hydrogen bond networks near proteins [24]. A series of $\mathrm{THz}$ spectroscopic studies $[11,26]$ on the water molecules near intrinsically-disordered AFGP and hyperactive antifreeze protein (hAFP) provide new clues to understanding the mechanisms of the antifreeze activity. It was revealed that a long-range effect on the hydration water dynamics near AFGP can extend to a dynamical hydration shell up to $\sim 20 \AA$ at $20{ }^{\circ} \mathrm{C}$ and $\sim 35 \AA$ at $5{ }^{\circ} \mathrm{C}$, indicative of a fully perturbed water network [26]. Upon investigation, the complexation reaction between the bindingactive hydroxyl group of AFGP and borate showed a reduction in the long-range water interaction accompanied by a significant decrease of the antifreeze activity. For hAFP, it was found that the extended hAFP-water network contributes to the antifreeze hyperactivity and the addition of the osmolyte sodium citrate increases both the size of the dynamical hydration shell and the antifreeze activity, a correlation between the longrange hydration dynamics and the antifreeze activity. Similar trends were also spotted in 
molecular dynamics simulations [28]. Water molecules near the binding sites were found to be significantly slower than those in the bulk and the retardation shifts the vibrational density of states of the hydration water to a higher frequency region between 2.4 and 2.7 $\mathrm{THz}$, indicative of a stronger hydrogen bonding environment. The blue shift leads to an increase in the vibrational density of states (VDOS) above $1.5 \mathrm{THz}\left(50 \mathrm{~cm}^{-1}\right)$ and a decrease of VDOS in the low frequency range, which can be probed experimentally [22].

Overall, the $\mathrm{THz}$ spectroscopic studies reveal that, although different types of AFP may have different mechanisms for antifreeze activity, there are at least two mechanisms that contribute to the antifreeze activity: a) a local, short-range interaction between the AFP and water, such as hydroxyl bond formation by threonine which causes enthalpic stabilization and b) a collective long-range interactions between the AFP and a sizable hydration layer which minimizes the entropic cost upon binding. These studies were shedding new light on perhaps one of the most difficult recognition problems in molecular biology [13].

AFPs may make use of either mechanism or both, we proposed that the shortrange interactions determine the affinity for docking of the protein surface to nanoscale ice crystals (thus preventing further growth). By a combination of experimental and simulation studies, we found an extended dynamical hydration shell including water molecules that shows retardation in the hydrogen bond network dynamics [11,12,26,30], in agreement with further theoretical model predictions by Matyushov [14]. The retardation of the hydration dynamics near the docking site is decreasing the entropy change upon desolvation and binding to a nano-ice crystal.

The retardation of hydration bond dynamics at the docking site was confirmed by 
recent Sum Frequency Generation (SFG) studies that reported an IR signature of the protein-water interface indicating ice-like water dynamics [15]. This is the first time that clear evidence for the presence of ice-like water layers at the ice-binding site of the protein in aqueous solution at temperatures above the freezing point was found. Decreasing the temperature to the biological working temperature of the protein $\left(0{ }^{\circ} \mathrm{C}\right.$ to $-2{ }^{\circ} \mathrm{C}$ ) increased the amount of ice-like water, while a single point mutation in the icebinding site was observed to completely disrupt the ice-like character and to eliminate antifreeze activity, indicating that the ordered ice-like water layers are responsible for the protein recognition and binding to ice.

Here we provide more evidence of the balance between short and long-range interactions in the anti-freeze activity.of AFP-III which can be tuned by co-solutes. The present work focuses on the AFP-III proteins purified either from the blood of antarctic eelpout Pachycara brachycephalum or acquired from A/F protein company. Unlike AFPI, AFP-III does not have a repeating ice-binding plane but a binding site that helps the globular structure occupy a niche in the ice surface, reflecting the intrinsic structural complexity in the antifreeze activity that evolution develops.

\section{MATERIALS AND METHODS}

\section{Experimental}

Type III AFP was purified from the eel pout. Sodium citrate was purchased from Sigma-Aldrich (St. Louis, MO) and used without further purification. Sample solutions were prepared using fresh ultra-pure Milli-Q water. Antifreeze activity was determined using a Clifton Nanoliter Osmometer as described elsewhere [11]. Terahertz absorption 
spectroscopy measurements were carried out using a p-Germanium difference laser spectrometer as described before $[11,12]$. We have recorded the change of collective protein-water network motions as induced by the AFP-III by measuring the concentration dependent changes in the transmitted $\mathrm{THz}$ radiation using bulk water as a reference.

Via a beam splitter THz pulses have been transmitted through AFP-III sample solutions and the reference cell (filled with water) and the transmission was recorded by a bolometer detector. Both cells were kept at identical conditions: $\mathrm{THz}$ absorbance measurements were performed at low-humidity $(<8 \%)$ and under temperature stabilized conditions $(\Delta T= \pm 0.05 \mathrm{~K})$.

Based upon the measurements, we determined the difference $\Delta \alpha$ between the integrated $\mathrm{THz}$ absorbance of the AFP-III sample solution $\left(\alpha_{\text {sample }}\right)$ and of the aqueous reference $\left(\alpha_{\text {reference }}\right)$ in the frequency range between 2.4 and $2.7 \mathrm{THz}$ as: $\Delta \alpha=\alpha_{\text {sample }}$ $\alpha_{\text {reference. }}$ as a function of protein concentration.

We find, for the AFP-III solvated in water and the AFP-III solvated in $0.5 \mathrm{M}$ sodium citrate, an almost linear decrease while for the concentration above $5 \mathrm{mg} \mathrm{ml}^{-1}$ a $\mathrm{THz}$ absorption that is higher than the linearly extrapolated linear decrease. In both cases, $\Delta \alpha$ was plotted, e.g., the THz absorption coefficient of the solvated protein versus the THz absorption of the buffer (water or solvated sodium citrate).

As a control, we also have recorded the influence of adding $0.5 \mathrm{M}$ sodium citrate to the water. We find that upon addition, the THz absorption is increased by $\Delta \alpha=40 \mathrm{~cm}^{-1}$. A considerable increase in $\mathrm{THz}$ absorption has also been found before upon addition of salts and amino acids $[16,17]$ 


\section{Simulation}

For the MD simulation, the starting structure of AFP-III and the mutant A16H (Ala16 -> His) were obtained from the Protein Data Bank (PDB ID: 1MSI and 3MSI, respectively). Both are from the ocean eel pout Macrozoarces americanus, which has the same antifreeze activity as the Antarctic eel pout Pachycara brachycephalum and quite similar sequence with more than $65 \%$ alignment score by the CLUSTER 2.1 multiple sequence alignment program. Constraints were applied to all covalent bonds including hydrogen atoms with the SHAKE algorithm. Periodic boundary conditions were used.

All MD simulations were performed in the canonical (NPT) ensemble with the GROMACS version 4.5 software package [18]. In each system, the protein was minimized for 5000 steps with steepest descent algorithm using AMBER03 force field [19], after its solvation in a $60 \AA$ A cubic water box of approximately 6700 TIP5P [20] water molecules (the $0.5 \mathrm{M}$ sodium citrate solution system has 65 citrate anions and 195 sodium ions, and approximately 6000 TIP5P water molecules). Subsequently the system was equilibrated for 400 ps. For the first 100 ps the system was restrained before it was released for 300 ps. Following the equilibration, trajectories of 5 ns were obtained at 300 $\mathrm{K}$ and $250 \mathrm{~K}$, respectively, with a Nosé-Hoover thermostat. Non-bonded interactions were gradually brought to zero by a shift function for electrostatic interaction as well as a switch function for van der Waals interactions between 10 and $12 \AA$. All simulations were carried out by integrating Newton's equations of motion with the Verlet algorithm using a $1 \mathrm{fs}$ time step. The system coordinates and velocities were stored every $10 \mathrm{fs}$. The hydrogen bond lifetime correlation function, $C_{\mathrm{HB}}(t)$, was computed for hydrogen bonds between water molecules and the protein at $300 \mathrm{~K} . C_{\mathrm{HB}}(t)$ is defined as the probability 
that, if a hydrogen bond between donor, $\mathrm{D}$, and acceptor, A, exists at $t=0$, then it still exists at time, $t$, even if the bond breaks at some intermediate time. We adopt a standard criterion for hydrogen bonds, i.e., a D-A distance of $3.5 \AA$ and a D-H-A angle greater than $150^{\circ}$ [21]. In our previous paper [22], we showed that retardation in hydration bond dynamics of water is correlated with a blue shift in the vibrational density of states for the hydrogen bond network modes. Experimentally, this could be observed as an increase in $\mathrm{THz}$ absorption above $50 \mathrm{~cm}^{-1}$ and a decrease in $\mathrm{THz}$ absorption below $50 \mathrm{~cm}^{-1}$. In the frequency range considered, we expect an intensity increase upon retardation of hydrogen bond dynamics.

\section{RESULTS AND DISCUSSION}

\section{Experimental}

In order to evaluate the AFP-III activity we perform freezing point hysteresis measurements using a Clifton Nanoliter Osmometer. Figure 1 shows the results of the freezing point hysteresis of AFP-III in water and in $0.5 \mathrm{M}$ sodium citrate. The measurements show a two-fold increase of activity upon addition of sodium citrate. The hysteresis describes the difference between melting and freezing temperature point and is a measure of antifreeze activity. The onset of the saturation of the activity takes place in both cases at a concentration of ca. $5 \mathrm{mg} / \mathrm{ml}$. 


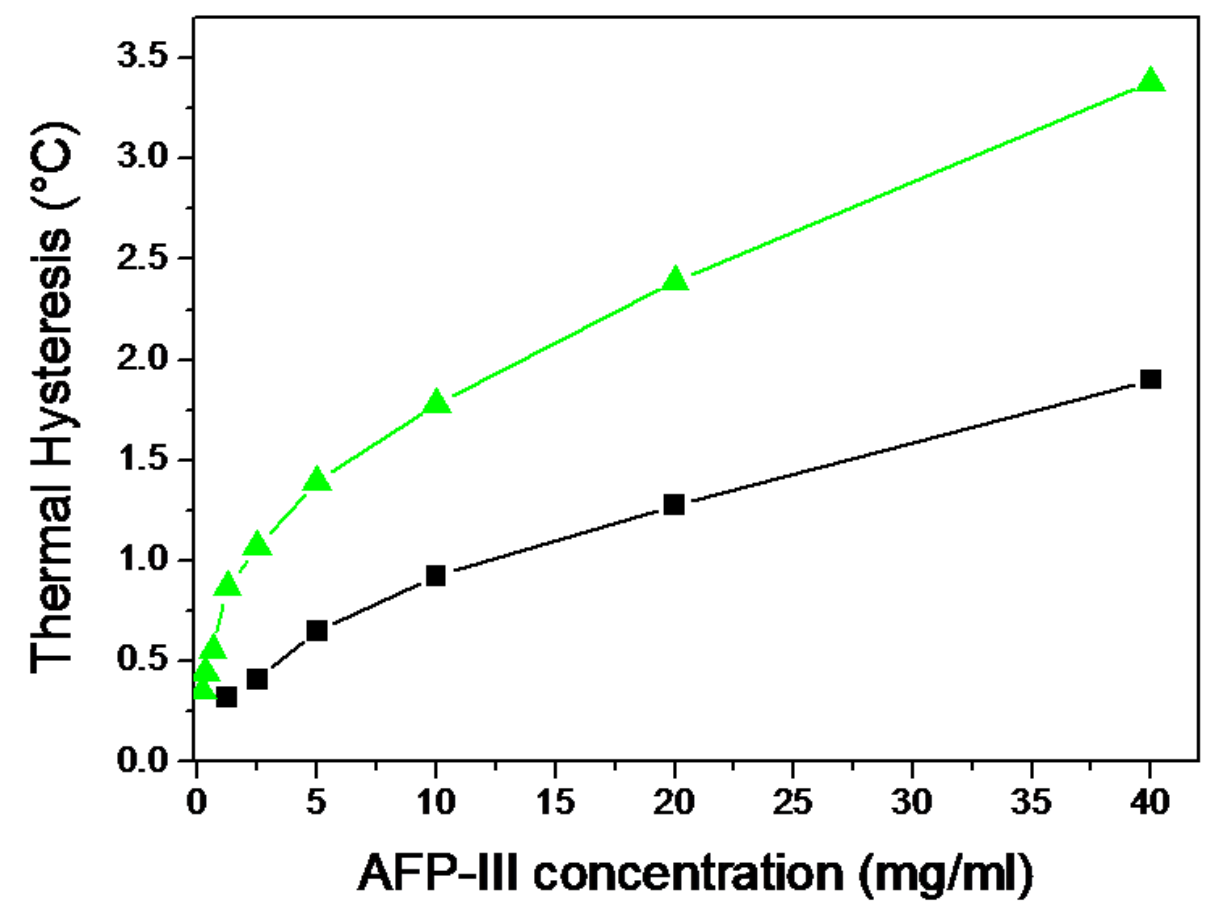

Figure 1: Freezing point hysteresis of AFP-III in water $(\cdot)$ and in $0.5 \mathrm{M}$ sodium citrate $(\triangle)$ determined using a Clifton Nanoliter Osmometer.

In previous measurements on DAFP-1, we have discovered a significant influence of the co-solutes on the hydrogen bond dynamics $[11,23]$. The discovery motivates us here to investigate the effect of addition of sodium citrate on the hydrogen bond network dynamics for a different class of AFP via terahertz absorption measurements and molecular dynamics simulations. In particular, we have previously shown that the retardation of the hydrogen bond dynamics in the hydration shell of DAFP-1 upon the addition of co-solutes is correlated with an increase of $\mathrm{THz}$ absorption in the frequency range between 2.4 and $2.6 \mathrm{THz}[22,23]$, a property we have referred as the "THz excess" [24]. This was found to be a necessary condition for activity; however, the magnitude of 
the activity did not seem to be correlated with the size of the THz effect.

Here we recorded the absorption coefficient of solvated AFP-III using our pGermanium THz absorption difference spectrometer [25]. Either bulk water or a sodium citrate buffer serves as reference. In Fig. 2, we display the change of the absorption coefficient of the sample compared to buffer reference as a function of protein concentration. Experimentally, we observe for higher protein concentration a small increase in THz absorption compared to the buffer. For AFP-III the increase in absorption coefficient compared to the predicted decrease in $\mathrm{THz}$ absorption by the $\mathrm{THz}$ excess is small compared to the observed change for DAFP [11,23].

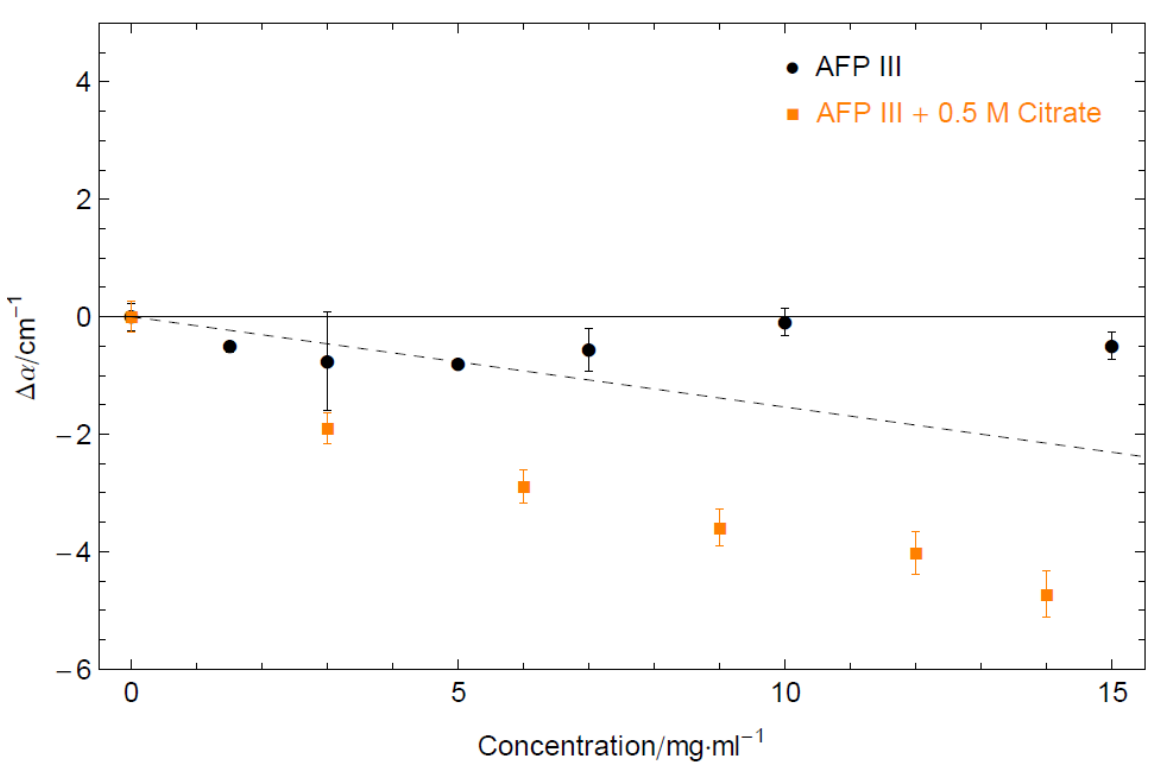

Figure 2: Left: Concentration-dependent integrated terahertz absorption of AFP-III solvated in water (black) and in $0.5 \mathrm{M}$ sodium citrate (red) at $293.15 \mathrm{~K}\left(20^{\circ} \mathrm{C}\right) \pm$ $0.5 \mathrm{~K}$. Plotted is the difference in $\mathrm{THz}$ absorption in comparison to buffers integrated in the frequency range from 2.4 to $2.7 \mathrm{THz}$. 
The addition of $0.5 \mathrm{~mol}$ sodium citrate to the solvated protein results in a significant decrease in $\Delta \alpha$ beyond the experimental uncertainty, which is at first glance surprising. If we take a closer look we see that the concentration dependent $\mathrm{THz}$ absorption coefficient of AFP-III upon the addition of sodium citrate follows first a linear decrease by about $4 \mathrm{~cm}^{-1}$ at $5 \mathrm{mg} \mathrm{ml}^{-1}$ and for higher protein concentrations we observe a nonlinear behavior and an excess compared to the linear extrapolation of the first two data points. All data points are obtained using sodium citrate as a reference; therefore we have investigated the effect of adding sodium citrate to bulk water. We have recorded the change in $\mathrm{THz}$ absorption when adding sodium citrate to water.

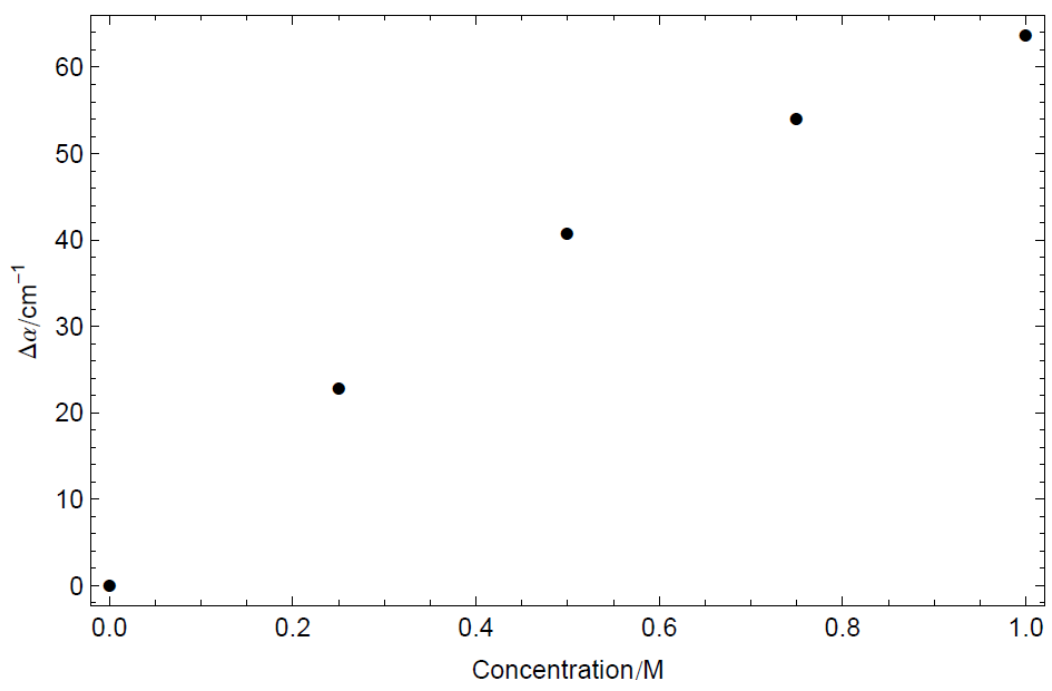

Figure $3 \mathrm{THz}$ absorption of solvated sodium citrate compared to water as a function of sodium citrate concentration.

We observed a linear increase in $\mathrm{THz}$ absorption when increasing the concentration of sodium citrate. The increase compared to water amounts to $40 \mathrm{~cm}^{-1}$ at $0.5 \mathrm{mg} / \mathrm{ml}$.

This increase for solvating charged species has been observed before and could be 
explained by the low frequency spectrum of solvated ions and anions, which exhibit so called rattling modes or translational modes between 50 and $400 \mathrm{~cm}^{-1}$, i.e. correlated and anti-correlated motions of the charges with the hydration cage. [26]

For a protein concentration of zero this implies that $\Delta \alpha$ would be increased by $\Delta \Delta \alpha=40$ $\mathrm{cm}^{-1}$ if we take water instead of a sodium citrate as a reference. How can we explain the observed decrease of $\Delta \alpha=\alpha$ (protein + sodium citrate + water) $-\alpha$ (sodium citrate + water) with increasing protein concentration? There are two ways:

If each protein, as well sodium citrate would be separated by several hydration shells and would have their own separated solvation shell, then the response would be strictly additive and $\Delta \alpha$ would be a measure for the solvated protein only. However, if sodium citrate binds to the protein or when both share their hydration shells, we would expect a non additive response. In the extreme case, all sodium citrate would be bound to the protein. As a consequence, the increase of $\mathrm{THz}$ absorption, due to the rattling mode of the sodium or the citrate with its hydration cage, would be suppressed.. The latter effect is protein specific and depends on the number of sodium or citrate ions that are actually bound to the protein surface and thus be proportional to the protein concentration. Here, we attribute the observed linear decrease for small protein concentrations- by an otherwise unchanged nonlinear behavior- to the suppression of the free motion of fully solvated sodium and citrate ions. The maximum decrease is expected when all sodium citrate ions are bound to the surface, thus we expect a maximum decrease in absorption by $40 \mathrm{~cm}^{-1}$. We thus initially estimate that about $10 \%$ are bound.

The addition of cosolutes is accompanied by an enhancement of the antifreeze activity. We have proposed that retardation of hydrogen bond dynamics is a necessary 
condition for antifreeze activity. In order to test this hypothesis on a molecular scale we have carried out accompanying MD simulations.

\begin{abstract}
Simulation
To examine protein-water dynamics at the interface, we have calculated lifetimes of the hydrogen bonds between AFP-III and water by MD simulations. In Figure 3, we show the hydrogen bond lifetime correlation function $C_{\mathrm{HB}}(t)$ for water molecules around AFP-III in aqueous and sodium citrate solution.
\end{abstract}




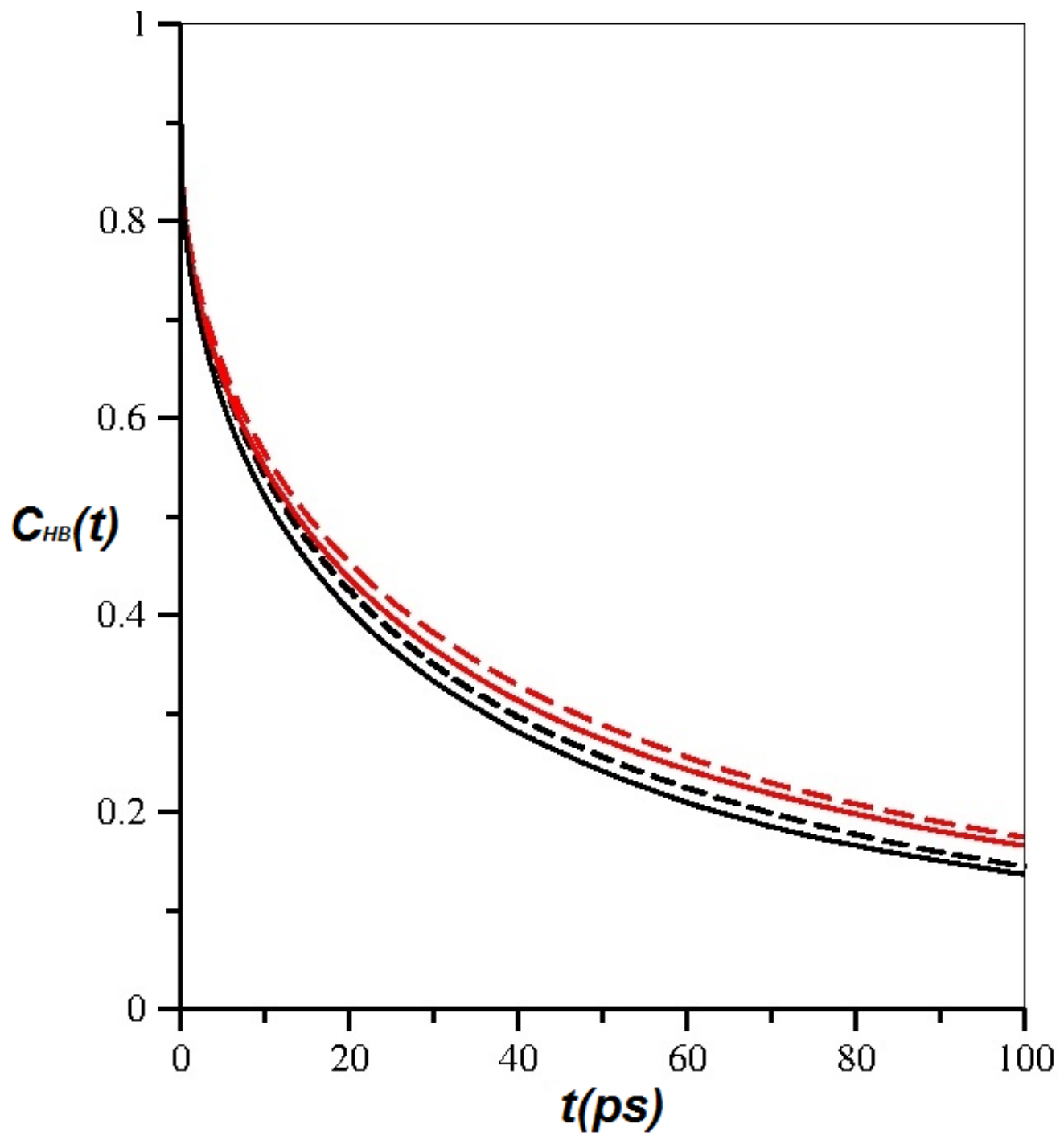

Figure 4: Hydrogen bond lifetime correlation functions $C_{\mathrm{HB}}(t)$ for water molecules of the AFP-III in the sodium citrate solution (dashed red) and aqueous solution (solid red), and the AFP-III mutant in the in the sodium citrate solution (dashed black) and aqueous solution (solid black), all at $300 \mathrm{~K}$.

Both hydrogen bond correlation functions do not follow a single exponential decay. In the presence of sodium citrate the simulations predict an increase in hydrogen 
bond lifetimes, similar to the general trend observed in a previous study of DAFP-1 $[11,23]$. At $300 \mathrm{~K}$ we observe that $C_{\mathrm{HB}}(t)$ reaches a value of 0.2 for hydrogen bonds between water and the mutant protein - which has a decreased antifreeze activity- after $61 \mathrm{ps}$ in aqueous solution, compared to $84 \mathrm{ps}$ for wild-type protein in sodium citrate solution. These values can be compared to the value of 23 ps at $300 \mathrm{~K}$ for bulk water [21]. For DAFP-1 [11], which has a well-defined ice-binding site, $C_{\mathrm{HB}}(t)$ reads 0.2 at 72 ps for hydrogen bonds between water and the ice-binding site, and 45 ps at the nonbinding site. For DAFP-1, the typical hydrogen bond lifetime is increased from 72 ps to 90 ps at the ice-binding site and from 46 ps to 66 ps at the non-ice-binding site upon the addition of sodium citrate. This suggests a similar hydrogen bond behavior for two-types of antifreeze proteins.

To gain more insights into the influence of AFP-III on hydrogen bond dynamics in the dynamic hydration shell, we address specifically the question of how the hydration dynamics are affected by mutations in the vicinity of the binding site. Here, a conserved alanine (Ala16) in the center of the planar surface, which is believed to be remarkably flat and to function as ice-binding surface, is substituted with a large residue His to disrupt the surface planarity and to sterically block the ice formation with $75 \%$ loss of antifreeze activity as determined experimentally $[27,28]$. The simulated hydrogen bond correlation function, plotted in Fig. 3, reveals that the water around the ice-binding site of the mutant is much more mobile than in the wild-type. These results show that even a single mutation can cause a noticeable difference of the hydrogen bond dynamics near the icebinding site. This is even more striking than the results of a previous computational study of a different AFP, where 4 point mutations affect the hydrogen bond dynamics [29]. Our 
observations here further support our hypothesis that the gradient of hydrogen bond dynamics towards the ice binding site - a "hydration funnel"- is correlated with antifreeze activity. We should also mention that observations in our simulations are consistent with earlier studies on the same type of AFP by various techniques [30,31].

The addition of sodium citrate has similar influence on the hydrogen bond dynamics near the binding sites in both wild-type and mutant, regardless of the antifreeze activity. This points towards a more local mechanism which determines the actual size of the antifreeze activity, different from what we have observed in other types of the same antifreeze protein family but similar to the 37 -residue $\alpha$-helical type I AFP from the winter flounder $[11,12,15,23,30,32]$. It seems that the local binding affinity is affected by the addition of sodium citrate. In the paper by Davies et al. [29], a short-range enthalpic interaction was proposed: the water molecules at ice-binding sites are thought to be anchored into an ice-like lattice docking via hydrogen bonds with an increased affinity to the ice crystal, which might be the determining factor for antifreeze activity. As discussed, the decrease in $\mathrm{THz}$ absorption points towards a direct interaction of the sodium citrate with the protein surface. Thus, this interaction might lead to a strengthening of the hydrogen bonding.

\section{CONCLUDING REMARKS}

Previously, we proposed two distinct mechanisms for antifreeze activity which can assist or enhance AF activity based on $\mathrm{THz}$ spectroscopy measurements $[11,12,15,23,30,32]:$ 
a) A short range local enthalpic interaction depending on the positioning of the threonine in order to form strong hydroxyl bonds with the $\mathrm{OH}$ of water molecules

b) A long range interaction via protein-induced water dynamics extending several hydration shells from the protein surface.

Both the interactions contribute to the free energy of the antifreeze activity. Whereas this leads to an increase in enthalpy upon binding, the latter decreases the entropic cost associated with binding. The long-range gradient can cause a preference for ice nanocrystals to drift towards the ice binding site due to a decreased entropic cost. For AFGP we found that the second mechanism dominates: antifreeze activity is correlated with the observation of a long range interaction by the influence of the protein on the collective hydrogen-bond dynamics: The addition of co-solutes such as sodium citrate causes a more pronounced hydrogen-bond gradient dynamics between the ice-binding site and ice, and is also correlated with a more efficient antifreeze activity without changing the structure or the positioning of the threonines [11].

However, for AFP-III the magnitude of the antifreeze activity depends mainly on the short range local binding [30]. Here, sodium citrate seems to directly interact with the protein and will thus effect the antifreeze activity.

For AFP- III the long-range perturbation of the protein hydration shell is less pronounced than for DAFP (as indicated by a smaller THz excess). However, the gradient in the hydrogen bond dynamics may still assist the antifreeze activity of wf-AFP-I, though these are probably not the only or the main mechanism for the antifreeze activity.

Generally, Nature follows (at least) two distinct molecular mechanisms to prevent freezing in cells. The most efficient AFPs can combine several strategies. 


\section{ACKNOWLEDGMENTS}

This work was initiated by funding of the Volkswagen Stiftung (M.H. and D.M.L.). Additional funding was provided by Ruhr-Universität Bochum (K.M. and M.H.) and by NSF CHE-1361776 (D.M.L.). We thank Shivang Vachharajani and Michael Senske for running gel electrophoresis of the protein. C. G. Bischak acknowledges funding from the U.S. Department of State Fulbright U.S. Student Program Grant. This work is supported by the Cluster of Excellence RESOLV (EXC 1069) funded by the Deutsche Forschungsgemeinschaft.

\section{REFERENCES}

[1] F.P. Miller, A.F. Vandome, J. McBrewster, Antifreeze protein, Alphascript Publishing, 2010.

[2] D.L. Denlinger, R.E.L. Jr, Low Temperature Biology of Insects, Cambridge University Press, 2010.

[3] S.P. Graether, Biochemistry and Function of Antifreeze Proteins, Nova Science Pub Incorporated, 2011.

[4] B. Moffatt, V. Ewart, A. Eastman, Physiol. Plant. 126 (2006) 5.

[5] L. Pham, R. Dahiya, B. Rubinsky, Cryobiology. 38 (1999) 169.

[6] A. Jorov, B.S. Zhorov, D.S.C. Yang, Protein Sci. 13 (2004) 1524.

[7] J.G. Duman, A.L. DeVries, Comp. Biochem. Physiol. Part B Comp. Biochem. 54 (1976) 375.

[8] E. Kristiansen, K.E. Zachariassen, Cryobiology. 51 (2005) 262.

[9] C.P. Garnham, R.L. Campbell, P.L. Davies, Proc. Natl. Acad. Sci. U. S. A. 108 (2011) 7363.

[10] A.D.J. Haymet, L.G. Ward, M.M. Harding, C.A. Knight, FEBS Lett. 430 (1998) 301.

[11] K. Meister, S. Ebbinghaus, Y. Xu, J.G. Duman, A. DeVries, M. Gruebele, et al., Proc. Natl. Acad. Sci. 110 (2013) 1617.

[12] S. Ebbinghaus, S.J. Kim, M. Heyden, X. Yu, U. Heugen, M. Gruebele, et al., Proc. Natl. Acad. Sci. 104 (2007) 20749.

[13] K.A. Sharp, Proc. Natl. Acad. Sci. 108 (2011) 7281.

[14] D.N. LeBard, D.V. Matyushov, J. Phys. Chem. B. 114 (2010) 9246.

[15] K. Meister, S. Strazdaite, A.L. DeVries, S. Lotze, L.L.C. Olijve, I.K. Voets, H.J.Bakker, Proc. Natl. Acad. Sci. 111 (2014) 17732. 
[16] S. Funkner, G. Niehues, D.A. Schmidt, M. Heyden, G. Schwaab, K.M. Callahan, et al. J. Am. Chem. Soc. 134 (2012) 1030.

[17] J. Sun, G. Niehues, H. Forbert, D. Decka, G. Schwaab, D. Marx, et al., J. Am. Chem. Soc. 136 (2014) 5031.

[18] S. Pronk, S. Páll, R. Schulz, P. Larsson, P. Bjelkmar, R. Apostolov, M.R. Shirts, J.C. Smith, P.M. Kasson, D. van der Spoel, B. Hess, E. Lindahl, Bioinforma. Oxf. Engl. 29 (2013) 845.

[19] E.J. Sorin, V.S. Pande, Biophys. J. 88 (2005) 2472.

[20] M.W. Mahoney, W.L. Jorgensen, J. Chem. Phys. 112 (2000) 8910.

[21] A. Luzar, D. Chandler, Nature. 379 (1996) 55.

[22] M. Heyden, M. Havenith, Methods. 52 (2010) 74.

[23] Meister, K., J.G. Duman, Y. Xu, A.L. DeVries, D.M. Leitner, M. Havenith, J. Phys. Chem. B. 118 (2014) 7920.

[24] D.M. Leitner, M. Gruebele, M. Havenith, HFSP J. 2 (2008) 314.

[25] A. Bergner, U. Heugen, E. Bründermann, G. Schwaab, M. Havenith, D.R. Chamberlin, et al., Rev. Sci. Instrum. 76 (2005) 063110.

[26] D.A. Schmidt, Ö. Birer, S. Funkner, B.P. Born, R. Gnanasekaran, G.W. Schwaab, et al., J. Am. Chem. Soc. 131 (2009) 18512

[27] C.I. DeLuca, P.L. Davies, Q. Ye, Z. Jia, J. Mol. Biol. 275 (1998) 515.

[28] C.P. Garnham, A. Natarajan, A.J. Middleton, M.J. Kuiper, I. Braslavsky, P.L. Davies, Biochemistry (Mosc.). 49 (2010) 9063.

[29] Y. Xu, R. Gnanasekaran, D.M. Leitner, J. At. Mol. Phys. (2012) e125071.

[30] S. Ebbinghaus, K. Meister, M.B. Prigozhin, A.L. DeVries, M. Havenith, J. Dzubiella, et al., Biophys. J. 103 (2012) L20.

[31] Meister, K., S. Lotze, L.L.C. Olijve, A.L. DeVries, J.G. Duman, I.K. Voets, H.J. Bakker, J. Phys. Chem. Lett. 6 (2015) 1162.

[32] S. Ebbinghaus, K. Meister, B. Born, A.L. DeVries, M. Gruebele, M. Havenith, J. Am. Chem. Soc. 132 (2010) 12210.

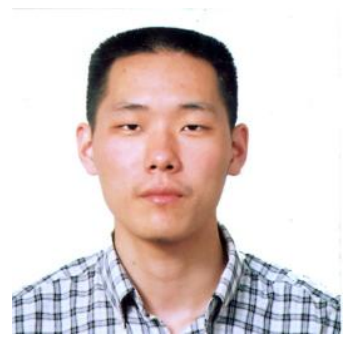


Yao Xu earned his B.Sc. in Applied Chemistry from Beijing University of Chemical Technology in P. R. China in 2006. He joined the doctoral program in Chemical Physics at the University of Nevada, Reno, U. S., where he obtained his Ph.D. under the supervision of Prof. David M. Leitner on the topic of solvation dynamics at the interface of proteins in 2013. Now he is a postdoctoral researcher with Prof. Martina Havenith at Ruhr University Bochum, Germany. He is interested in connecting solvation dynamics with biomolecular functions by molecular simulation and laser spectroscopy.

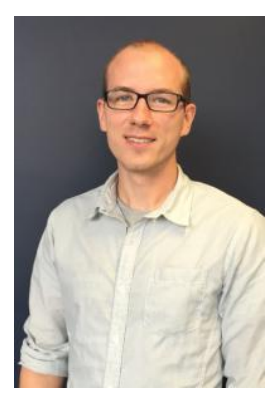

Alexander Bäumer was born in Dortmund, Germany in 1986. He received his B.Sc. and M.Sc. in biochemistry from the Ruhr University Bochum in 2011/2013. Currently he is a Ph.D. student in the laboratory of Prof. Martina Havenith at the Ruhr University Bochum. His research aims to gain understanding of solvation dynamics around biomolecules with the help of terahertz spectroscopy.

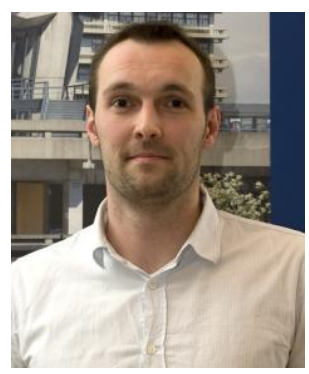

Konrad Meister was born in Herdecke, Germany. In 2013 he completed his PhD in physical chemistry at the Ruhr University Bochum, Germany. He is currently working as a Postdoctoral researcher at the FOM Institute AMOLF in Amsterdam where he was recently awarded a Marie Curie Scholarship. His research interests are the structure and hydration dynamics of biomolecular systems at interfaces.

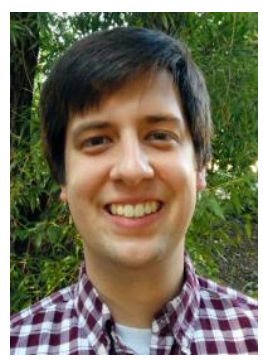


Connor G. Bischak was born in Redmond, Washington in 1987. He received a B.S. in chemistry from Haverford College in 2010 where he performed research with Casey Londergan. In 2011, Connor conducted research with Martina Havenith at the Ruhr Universität Bochum while on a Fulbright Fellowship. Currently, Connor is pursuing a $\mathrm{Ph} . \mathrm{D}$. in chemistry from the University of California, Berkeley under the supervision of Naomi Ginsberg, where his interests include developing new methods in electron microscopy and the nanoscale optical properties of emerging organic-inorganic solar cell materials.

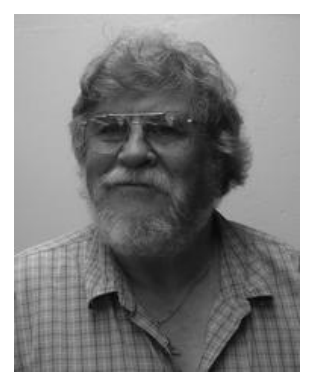

Art DeVries is a native of the state of Montana in the US. He received a degree in zoology at the University of Montana and a PhD in biology from Stanford University in 1968. He spent six years as a research associate in the Physiological Research Laboratory, Scripps Institute of Oceanography, and then accepted a position in the Department of Physiology at the University of Illinois, Urbana-Champaign. Presently he is a professor in the Department of Animal Biology and Molecular and Integrative Physiology. His research focus is in cold adaptation of polar fishes and is centered around understanding the role that antifreeze proteins play in their survival. His research interests include structure, function, origin and evolution of the antifreeze proteins.

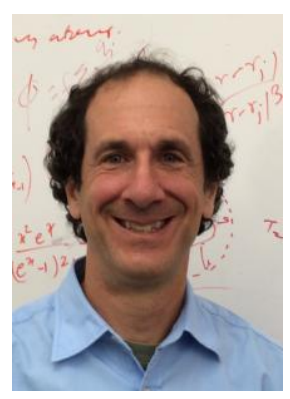

David Leitner received his B.S. in Chemical Engineering and B.A. in Chemistry at Cornell University and his Ph.D. in Chemical Physics at the University of Chicago working with Steve Berry. He did postdoctoral work with Jim Doll at Brown University, Lorenz Cederbaum at the University of Heidelberg, Germany, as an NSF postdoctoral fellow, and Peter Wolynes at the University of Illinois at Urbana-Champaign. Since 
2000 he has been at the University of Nevada, Reno, where he is Professor of Chemistry. Leitner is a Fellow of the American Physical Society and has served as Secretary/Treasurer of the Division of Chemical Physics. Areas of research include theoretical and computational studies of energy flow in molecules and its influence on chemical reaction kinetics, thermal transport on the nanoscale, and computational studies of terahertz spectroscopy and dynamics of solvated biomolecules.

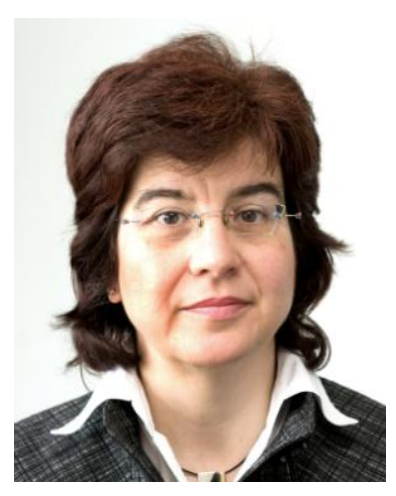

Martina Havenith studied physics and mathematics at the university in Bonn, Germany. She had several research stays at the UC Berkeley and at the Radboud University in Nijmegen. Since 1998 she holds a chair in physical chemistry at the Ruhr University Bochum. She is a member of the Leopoldina, the German Academy of Sciences. She is the director of the Center of the Molecular Spectroscopy and Simulation of Solvent Driven Processes in Bochum. 
Yao Xu earned his B.Sc. in Applied Chemistry from Beijing University of Chemical Technology in P. R. China in 2006. He joined the doctoral program in Chemical Physics at the University of Nevada, Reno, U. S., where he obtained his Ph.D. under the supervision of Prof. David M. Leitner on the topic of solvation dynamics at the interface of proteins in 2013. Now he is a postdoctoral researcher with Prof. Martina Havenith at Ruhr University Bochum, Germany. He is interested in connecting solvation dynamics with biomolecular functions by molecular simulation and laser spectroscopy.

Alexander Bäumer was born in Dortmund, Germany in 1986. He received his B.Sc. and M.Sc. in biochemistry from the Ruhr University Bochum in 2011/2013. Currently he is a Ph.D. student in the laboratory of Prof. Martina Havenith at the Ruhr University Bochum. His research aims to gain understanding of solvation dynamics around biomolecules with the help of terahertz spectroscopy.

Konrad Meister was born in Herdecke, Germany. In 2013 he completed his $\mathrm{PhD}$ in physical chemistry at the Ruhr University Bochum, Germany. He is currently working as a Postdoctoral researcher at the FOM Institute AMOLF in Amsterdam where he was recently awarded a Marie Curie Scholarship. His research interests are the structure and hydration dynamics of biomolecular systems at interfaces.

Connor G. Bischak was born in Redmond, Washington in 1987. He received a B.S. in chemistry from Haverford College in 2010 where he performed research with Casey Londergan. In 2011, Connor conducted research with Martina Havenith at the Ruhr Universität Bochum while on a Fulbright Fellowship. Currently, Connor is pursuing a Ph.D. in chemistry from the University of California, Berkeley under the supervision of Naomi Ginsberg, where his interests include developing new methods in electron microscopy and the nanoscale optical properties of emerging organic-inorganic solar cell materials.

Art DeVries is a native of the state of Montana in the US. He received a degree in zoology at the University of Montana and a PhD in biology from Stanford University in 1968. He spent six years as a research associate in the Physiological Research Laboratory, Scripps Institute of Oceanography, and then accepted a position in the Department of Physiology at the University of Illinois, Urbana-Champaign. Presently he is a professor in the Department of Animal Biology and Molecular and Integrative Physiology. His research focus is in cold 
adaptation of polar fishes and is centered around understanding the role that antifreeze proteins play in their survival. His research interests include structure, function, origin and evolution of the antifreeze proteins.

David Leitner received his B.S. in Chemical Engineering and B.A. in Chemistry at Cornell University and his Ph.D. in Chemical Physics at the University of Chicago working with Steve Berry. He did postdoctoral work with Jim Doll at Brown University, Lorenz Cederbaum at the University of Heidelberg, Germany, as an NSF postdoctoral fellow, and Peter Wolynes at the University of Illinois at Urbana-Champaign. Since 2000 he has been at the University of Nevada, Reno, where he is Professor of Chemistry. Leitner is a Fellow of the American Physical Society and has served as Secretary/Treasurer of the Division of Chemical Physics. Areas of research include theoretical and computational studies of energy flow in molecules and its influence on chemical reaction kinetics, thermal transport on the nanoscale, and computational studies of terahertz spectroscopy and dynamics of solvated biomolecules.

Martina Havenith studied physics and mathematics at the university in Bonn, Germany. She had several research stays at the UC Berkeley and at the Radboud University in Nijmegen. Since 1998 she holds a chair in physical chemistry at the Ruhr University Bochum. She is a member of the Leopoldina, the German Academy of Sciences. She is the director of the Center of the Molecular Spectroscopy and Simulation of Solvent Driven Processes in Bochum 


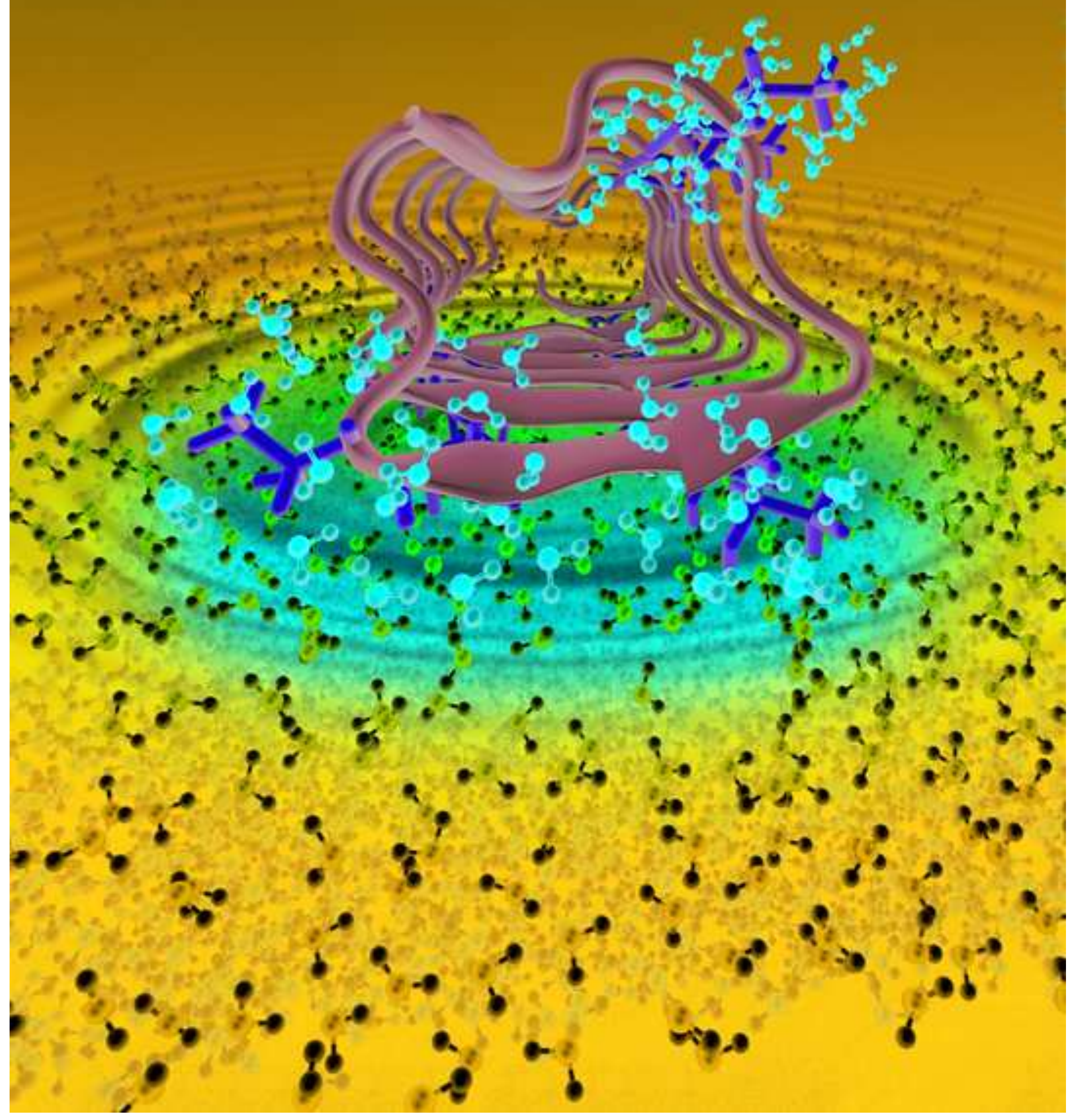

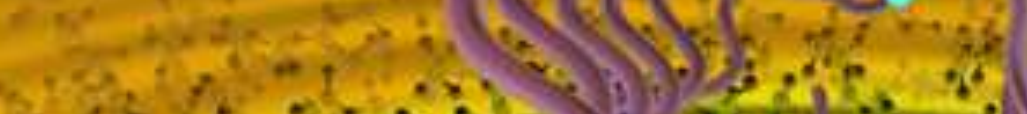

